\title{
Die differenzierte Therapie karpaler Bandverletzungen
}

\author{
Kai Megerle, Hans-Günther Machens
}

\section{Zusammenfassung}

Karpale Bandverletzungen sind komplexe Krankheitsbilder, die zu einer Instabilität der Handwurzel mit frühzeitiger Arthroseentwicklung führen. Am häufigsten ist dabei das skapholunäre Band zwischen Kahnbein und Mondbein betroffen. Diese Verletzungen werden nach wie vor häufig übersehen, müssen aber stadiengerecht diagnostisch aufgearbeitet und therapiert werden, um Spätfolgen zu vermeiden. Vor allem für die chronische skapholunäre Instabilität nach länger zurückliegenden Bandverletzungen sind zahlreiche konkurrierende Therapieoptionen beschrieben, die jedoch alle die ursprüngliche Biomechanik nicht wiederherstellen können. Der Artikel gibt einen Überblick über die differenzierte Diagnostik und Therapie der skapholunären Bandverletzung.

\section{Stage-specific treatment of carpal ligament injuries}

Injuries of the carpal ligaments are complex entities leading to carpal instability and, subsequently, premature degenerative arthritis of the radiocarpal joint. In most cases, the scapholunate ligament between the scaphoid and lunate bones is affected. Even today, injuries of these ligaments are often missed and treatment is delayed. However, scapholunate ligament injuries require meticulous diagnostic workup and treatment depending on injury stages to avoid late complications. In particular, there are a large number of treatment options for established, chronic scapholunate instability secondary to past ligament injuries. However, none of them has been proven to properly restore carpal kinematics. This article provides a brief overview of the diagnostic workup and treatment of scapholunate ligament injuries.

\section{Einleitung}

Die Bedeutung der stabilen Handwurzel

Die Behandlung von Bandverletzungen der Handwurzel (Karpus) ist aus verschiedenen Gründen komplex. Einerseits ist bereits die Diagnose schwierig, sodass diese verspätet, manchmal erst nach Jahren bzw. Jahrzehnten, gestellt werden kann. Andererseits sind selbst bei frühzeitiger Diagnostik die Behandlungsoptionen mit unbefriedigenden klinischen Ergebnissen verbunden. Der folgende Artikel soll einen Überblick über

OP-JOURNAL 2015; 31: 114-118

(c) Georg Thieme Verlag KG Stuttgart · New York DOI http://dx.doi.org/10.1055/s-0035-1546210 die differenzierte Therapie der häufigsten Verletzungen geben.

Verletzungen des skapholunären Bandes sind von besonderer Bedeutung, weil sie zu einer „Instabilität“ der Handwurzel führen. Die Handwurzel kann dabei die auf sie einwirkenden Kräfte nicht mehr adäquat verarbeiten [1]. Typischerweise ist dies mit einer Fehlstellung der Knochen untereinander verbunden, die $\mathrm{zu}$ unphysiologischen Belastungen der Gelenkflächen und damit langfristig zu einem vorzeitigen arthrotischen Verschleiß führt.

Das Auftreten von arthrotischen Veränderungen an der Speiche ist das Endstadium der karpalen Instabilität und wird als „scapholunate advanced collapse“ (SLAC) bezeichnet.
Ist dieser erst einmal eingetreten, bleiben lediglich Rettungsoperationen, wie etwa eine Entfernung der proximalen Handwurzelreihe oder Teilversteifungen.

\section{Biomechanik des Handgelenks}

\section{Anatomie}

Die eigentliche Handwurzel wird distal von Radius und Ulna aus 8 Handwurzelknochen gebildet, die anatomisch in 2 funktionell sehr unterschiedliche Reihen eingeteilt werden können. Die Knochen der körpernahen Handwurzelreihe, das Kahnbein, das Mondbein und das Dreiecksbein (Os triquetrum) sind dabei sehr beweglich zueinander, während die körperferne Handwurzelreihe funktionell als ein unbeweglicher Block betrachtet werden kann.

\section{Ringmodell nach Lichtman}

Die meisten derzeit üblichen therapeutischen Ansätze beruhen auf dem Ringmodell nach David Lichtman.

Hierbei wird die gesamte Handwurzel als ein unter Spannung stehender Ring betrachtet. Diese Spannung entsteht dadurch, dass die einzelnen Handwurzelknochen bei Belastung eine natürliche Tendenz zur Rotation aufweisen, die bei intaktem Bandapparat jedoch unterdrückt wird.

Aufgrund seiner natürlichen Form hat das Kahnbein dabei die Tendenz, unter Last eine Flexionsbewegung zur Palmarseite hin durchzuführen, im Gegensatz hierzu rotiert das Os triquetrum unter Last in Richtung der Streckseite. Die Knochen der proximalen Handwurzelreihe sind somit 2 gegensätzlichen Kräften ausgesetzt, die durch die interkarpalen Bandverbindungen zwischen den Knochen, also das skapholunäre und lunotriquetrale Band, aufgefangen werden (Abb. 1). 


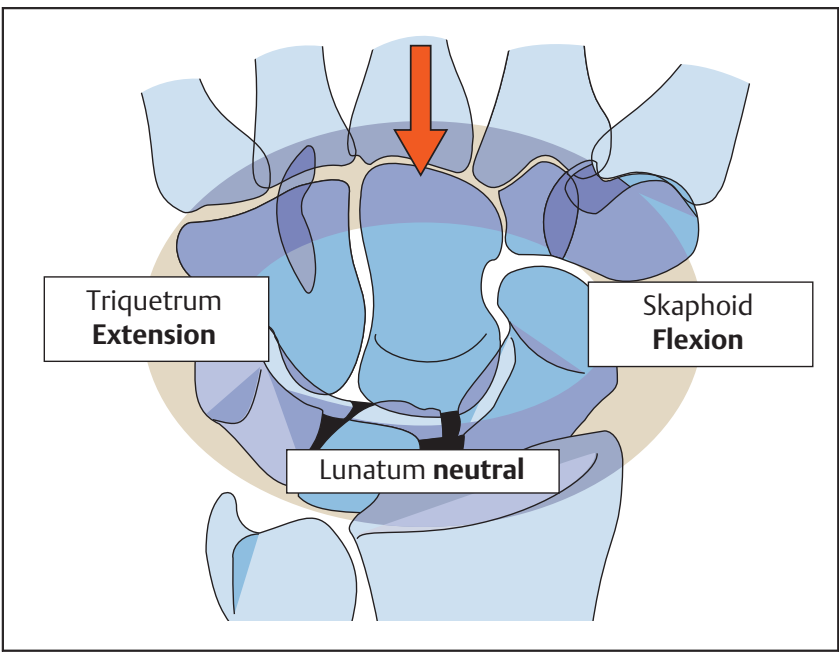

Abb. 1 Ringkonzept nach Lichtman. Die normale Handwurzel wird als unter Spannung stehender Ring betrachtet, in dem die Handwurzelknochen aufgrund der intakten Bandverbindungen nicht ihren natürlichen Tendenzen folgen können. Das Lunatum steht in Neutralstellung, da sich die flektierenden Kräfte des Skaphoids und die extendierenden Kräfte des Triquetrums ausgleichen.

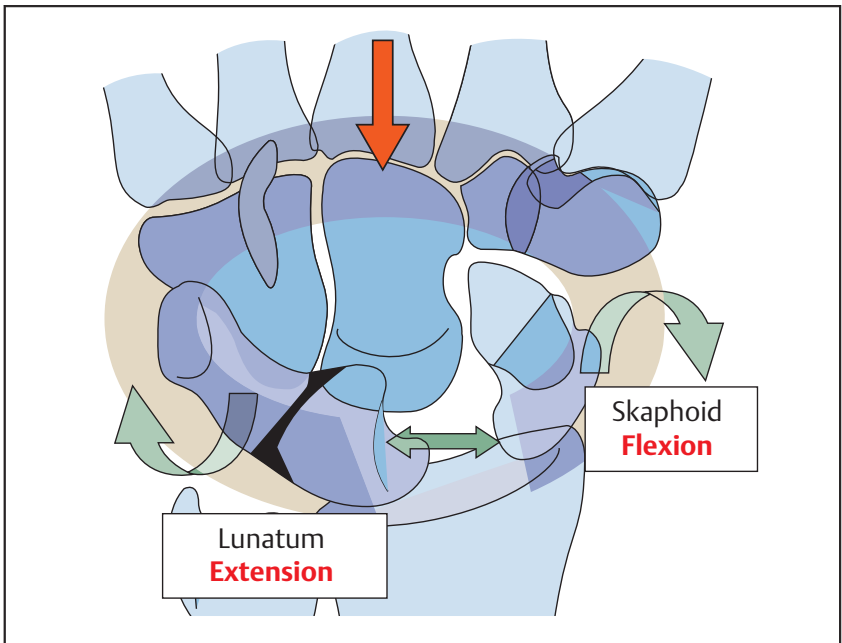

Abb. 2 Karpale Instabilität: Wird die Verbindung zwischen Skaphoid und Lunatum, z. B. durch eine skapholunäre Bandruptur, unterbrochen, folgen die karpalen Knochen ihren natürlichen Tendenzen. Bei intaktem lunotriquetralem Band folgt das Lunatum dem Triquetrum in die Extension, während das Skaphoid gegenläufig eine Pronationsbewegung durchmacht.
Erst wenn diese Spannung an einer Stelle unterbrochen wird, etwa durch eine Verletzung des skapholunären Bandes, können die Knochen ihrer natürlichen Tendenz folgen: das Skaphoid in die Flexion und das Os triquetrum in die Extension. Da das Mondbein über das intakte lunotriquetrale Band mit dem Os triquetrum verbunden ist, folgt es diesem in die Extension. Im Falle einer skapholunären Bandverletzung ergibt sich somit eine gegenläufige Rotation von Kahnbein und Mondbein, die im seitlichen Röntgenbild auffällig ist (Abb. 2) und als auch als DISI (dorsal intercalated segment instability) bezeichnet wird (Abb. 4).

Noch auffälliger ist das gleichzeitige Auseinanderweichen der Knochen in der posterioanterioren Röntgenprojektion. Dieses charakteristische Erscheinungsbild wird nach der berühmten Zahnlücke eines englischen Komikers auch als „,Terry-Thomas-Zeichen“ bezeichnet (Abb. 3).

\section{Diagnostik und Klassifikation}

Die Diagnose einer skapholunären Bandverletzung ist schwierig. Die Beschwerden sind bei akuten Verletzungen oft gering und werden bei ungenauer Untersuchung häufig als einfache „Handgelenksdistorsion“ fehldiagnostiziert. Tatsächlich bessern sich die Symptome häufig bereits nach kurzer Zeit, sodass eine weitere diagnostische Abklärung unterbleibt. Die Diagnose der Bandruptur wird daher oft verzögert gestellt, sodass eine direkte Bandnaht dann nicht mehr möglich ist. Besonders schwierig ist die Diagnose, wenn die Bandruptur in Kombination mit anderen Verletzungen, z.B. distalen Radiusfrakturen, auftritt. Die Häufigkeit dieser Kombinationsverletzungen wird mit bis zu 30\% angegeben [2].

\section{Klinische Untersuchung}

Nach der akuten Bandverletzung entsteht oft ein unspezifischer radiokarpaler Schmerz. Mit etwas Übung kann der skapholunäre Spalt jedoch gezielt palpiert werden.

Diagnostisch hilfreich kann zudem der Kahnbein-Verschiebetest nach Watson sein.

Hierbei wird bei Radialduktion der Hand das Skaphoid nach dorsal über die dorsale Radiuskante hinaus luxiert. Als pathologisch ist der Test jedoch nur bei einer deutlichen Seitendifferenz zu bewerten, falsch positiv kann er v.a. bei einer allgemeinen Bandlaxizität ausfallen.

\section{Bildgebende Untersuchung}

Grundlage der Diagnostik ist zunächst ein Röntgenbild des Handgelenks in 2 Ebenen. Zur Beurteilung der karpalen Winkel ist dabei die exakte Ausführung von großer Bedeutung. Für die Diagnose einer skapholunären Bandverletzung sind v.a. der Abstand zwischen Kahnund Mondbein sowie der Winkel zwi- schen den beiden Knochen im seitlichen Röntgenbild entscheidend [3]. In der Praxis sind diese Parameter variabel, sodass bei auffälligen Konfigurationen eine Vergleichsaufnahme der Gegenseite unbedingt zu empfehlen ist. Entscheidend für die Diagnose einer Instabilität sind der skapholunäre sowie der radiolunäre Winkel (Abb.4). In einer eigenen Analyse von über 800 Röntgenbildern deutete ein skapholunärer Winkel von $65^{\circ} \mathrm{mit}$ 95\%iger Wahrscheinlichkeit auf eine Verletzung des SL-Bandes hin [4]. Weiterhin können insbesondere bei dynamischen Instabilitäten Belastungsaufnahmen oder Kinematografien hilfreich sein [5].

Umstritten ist die Genauigkeit der kernspintomografischen Diagnostik außerhalb klinischer Studien. Falsch positive Befunde sind häufig, sodass diese Modalität, obwohl sehr häufig durchgeführt, für den weiteren Therapieverlauf nicht zwangsläufig erforderlich scheint [6]. Goldstandard der Diagnostik ist jedoch nach wie vor die Durchführung einer Handgelenksarthroskopie (Abb. 5).

\section{Einordnung der Instabilität}

Die skapholunäre Bandverletzung ist kein binäres Ja-/Nein-Phänomen, sondern kann eine Vielzahl verschiedener Ausprägungen aufweisen.

Entsprechend unterschiedlich ist auch der natürliche Verlauf. Einzelne Unterformen zeigen über viele Jahre z. B. kein 


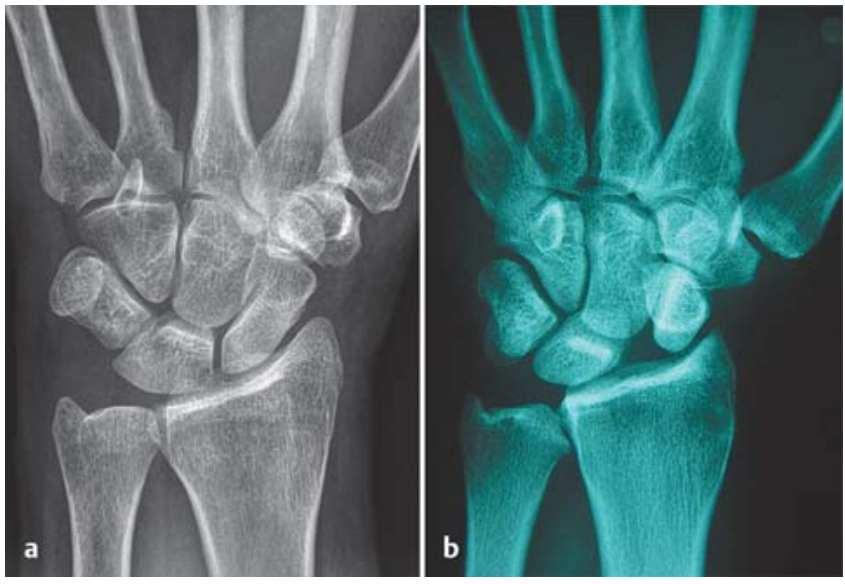

Abb. 3 Terry-Thomas-Zeichen. Links: Normalbefund, rechts: Durch Verletzung des skapholunären Bandes kommt es zu dem typischen Auseinanderweichen von Kahn- und Mondbein.

Voranschreiten der arthrotischen Degeneration [7]. Bei der Bestimmung der optimalen Therapie muss daher zunächst das Stadium der Instabilität festgelegt werden. In Anlehnung an M. Garcia-Elias et al. haben sich dabei folgende Fragen bewährt [8]:

1. Welche Symptome hat der Patient?

Die individuelle Beeinträchtigung in

Beruf und Freizeit spielt eine entscheidende Rolle bei der Wahl des Therapieverfahrens.

2. Liegt ein partieller oder kompletter Bandschaden vor?

Im Gegensatz zu kompletten Durchtrennungen haben partielle Bandschäden biomechanisch häufig keine oder nur geringe Auswirkungen.

3. Wie alt ist die Verletzung?

Primär nahtfähige Bandstümpfe, die eine direkte Naht ermöglichen, degenerieren rasch, sodass eine direkte Bandnaht nur bei frischen Verletzungen möglich ist.

4. Besteht eine Fehlrotation der Handwurzel nur unter Belastung?

Diese Frage unterscheidet die dynamische (nur unter Belastung auftretende) von der statischen (auch in Ruhe nachweisbaren) Instabilität.

5. Ist eine Reposition der Handwurzel möglich?

Ist eine Reposition nicht leicht zu erzielen, scheiden rekonstruktive Verfahren zur Wiederherstellung der ursprünglichen Anatomie aus und es sollte stattdessen eine Rettungsoperation angestrebt werden.

6. Ist der Gelenkknorpel bereits degenerativ verändert?

Auch bei bereits eingetretenen degenerativen Veränderungen des Gelenkknorpels sind rekonstruktive Verfahren nicht mehr indiziert.

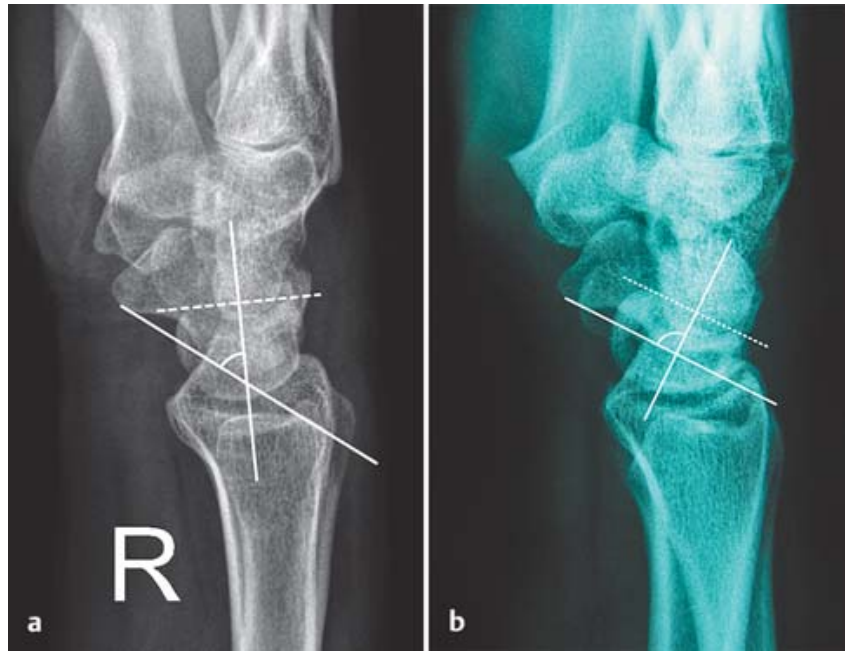

Abb. 4 DISI-Konfiguration im seitlichen Röntgenbild. Die Verletzung des skapholunären Bandes führt zu einer charakteristischen gegenläufigen Rotation von Kahnbein und Mondbein und damit zu einer Vergrößerung des skapholunären Winkels (links Normalbefund, rechts DISIStellung).
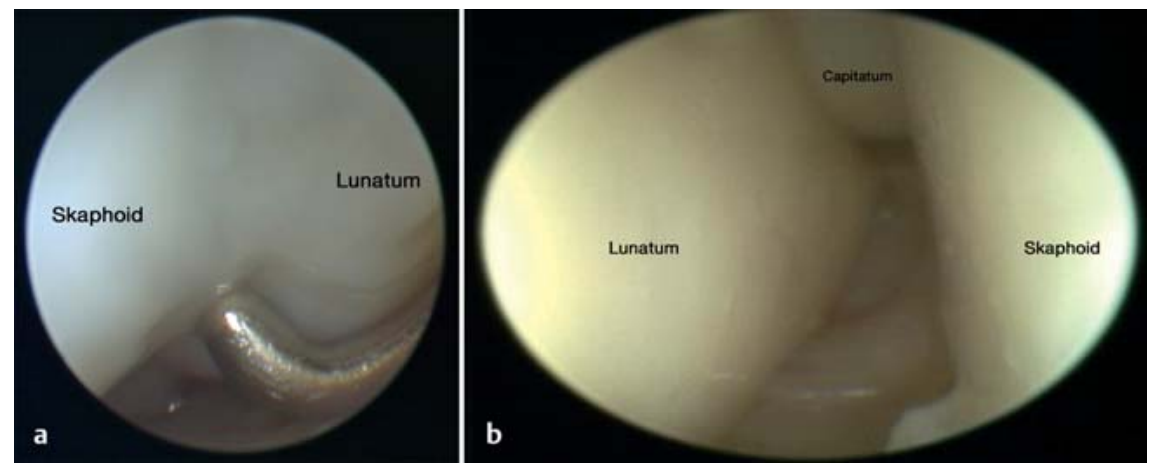

Abb.5 Arthroskopische Darstellung des skapholunären Bandes. Oben: Normalbefund, der Tasthaken demonstriert das intakte Band. Unten: Komplette SL-Bandruptur, es finden sich lediglich rudimentäre Bandreste, der Blick auf das Os capitatum ist frei.

Durch diese Fragen zum Therapiestadium lassen sich verschiedene klinische Situationen oder auch Krankheitsstadien unterscheiden. Im besten Fall liegt eine zufällig entdeckte partielle Ruptur des skapholunären Bandes vor, die nicht zu einer rotatorischen Instabilität der Handwurzel geführt hat (Stadium 1). Ein weiteres Szenario ist die frisch diagnostizierte, akute Bandruptur (Stadium 2), bei der eine direkte Bandnaht angestrebt werden sollte. Einen Streitfall stellen dynamische Instabilitäten (Stadium 3) dar. In dieser Patientengruppe kann eine vollständige Ruptur des skapholunären Bandes arthroskopisch gesichert werden. Wegen der intakten sekundären Stabilisatoren zeigen die Handwurzelknochen aber erst unter Belastung eine Fehlrotation. Am schwierigsten ist die Therapie von Patienten mit chronischer skapholunärer Instabilität (Stadium 4).
Nach wie vor existiert kein verlässliches Verfahren zur anatomiegerechten Rekonstruktion des skapholunären Bandes.

Daher sind alle derzeit üblichen Therapieoptionen eher als symptomatische Maßnahme zu betrachten, ohne dass ein Langzeiterfolg als gesichert gelten kann.

Ist eine Reposition des Karpus in die ursprüngliche Konfiguration nicht möglich bzw. zeigen sich bereits degenerativ veränderte Knorpeloberflächen im Bereich des Radiokarpalgelenks, so sind rekonstruktive Therapieansätze an der Handwurzel nicht mehr sinnvoll.

Diesen Patienten wird i.d.R. eine Rettungsoperation empfohlen. Im Wesentlichen bestehen hierbei 2 Optionen, nämlich die Entfernung der proximalen Handwurzelreihe oder die mediokarpale Teilarthrodese. Alternativ kann zur rein 
symptomatischen Verbesserung auch die Durchtrennung der Schmerznerven erfolgen. In vielen Fällen lässt sich dadurch eine gewisse Beschwerdereduktion für einen begrenzten Zeitraum erzielen.

\section{Therapieoptionen}

Für jedes Stadium der skapholunären Instabilität existiert eine Vielzahl unterschiedlichster Therapieoptionen. Diese reichen von einer konservativen physiotherapeutischen Behandlung über rein symptomatische Operationen wie Schmerznervendurchtrennungen bis $\mathrm{zu}$ Versteifungen bzw. Teilversteifungen des Handgelenks.

Die Wahl des Therapieverfahrens ist dabei häufig durch persönliche Erfahrung des Operateurs, weniger durch evidenzbasierte wissenschaftliche Daten bestimmt.

Die Optionen zur Behandlung der chronischen skapholunären Instabilität lassen sich erst nach einem Verlauf von mindestens 5 Jahren postoperativ überhaupt seriös einschätzen. Zudem sind viele der Operationen technisch anspruchsvoll und erfordern neben operativem Geschick auch ein gutes Verständnis der karpalen Biomechanik.

\section{Konservative Therapie}

Neuere biomechanische Studien haben gezeigt, dass die Stabilität des Carpus durch die über die Handwurzel hinwegziehenden Sehnen günstig beeinflusst werden kann. Diese Erkenntnisse sind Grundlagen eines neuen konservativen Therapiekonzepts, das derzeit an unserer Klinik in Erprobung ist. Andere Autoren empfehlen dagegen bereits frühzeitig operative Interventionen.

\section{Direkte Bandnaht}

Weitgehend unumstritten ist die Ansicht, dass bei nahtfähigen Bandstümpfen eine direkte Naht des skapholunären Bandes in Kombination mit einer Transfixation der Handwurzel angestrebt werden sollte [9]. Ungeklärt aber ist die Frage, wie lange diese Maßnahme sinnvoll ist. In unserer Praxis hat sich eine Bandnaht, die länger als 6 Wochen nach dem Unfallereignis erfolgt, als kaum praktikabel erwiesen.

Die Therapieoptionen zur Rekonstruktion eines nicht mehr direkt nahtfähigen

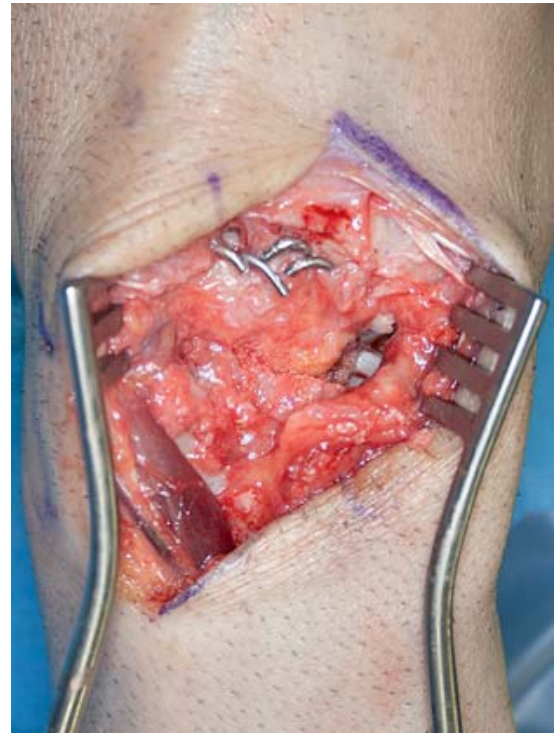

Abb. 6 Intraoperativer Situs der mediokarpalen Teilarthrodese. Das Skaphoid wurde entfernt und gibt den Blick auf die palmaren Handgelenksbänder frei. Capitatum, Lunatum, Hamatum und Triquetrum sind entknropelt und mittels 4 Kirschnerdrähten miteinander verblockt.

skapholunären Bandes lassen sich grundsätzlich in Weichteiloperationen (Kapsulodesen, Tenodesen), knöcherne Operationen (Bone-Ligament-Bone-Transplantationen, Teilarthrodesen) sowie Rettungsoperationen unterteilen. Wie die Vielzahl möglicher Therapieoptionen nahelegt, ist eine anatomiegerechte Rekonstruktion des skapholunären Bandes nicht möglich. Im Folgenden sollen nun die möglichen Therapieoptionen kurz in einem Überblick dargestellt werden.

\section{Arthrodesen}

Die skapholunäre Fusion wurde aufgrund der sehr unbefriedigenden klinischen Ergebnisse bereits frühzeitig verlassen [10]. Meist konnte angesichts der hohen mechanischen Belastungen im skapholunären Übergang keine stabile knöcherne Fusion erzielt werden und selbst Patienten mit erfolgreich fusionierten Handwurzelknochen wiesen eine sehr schlechte Beweglichkeit und hohe Schmerzhaftigkeit auf, sodass dieses Verfahren heutzutage als obsolet gilt. Eine nach wie vor gelegentlich praktizierte Variante stellt die temporäre Transfixation der beiden Knochen, etwa durch eine Herbert-Schraube dar (RASL procedure) [11]. Da die Gelenkachse zwischen diesen beiden Knochen durch die eingebrachte Schraube nicht exakt ist, kommt es auch in diesen Fällen häufig zu einer Lockerung des Implantats ohne ausreichenden biomechanischen Effekt.

\section{Kapsulodesen}

Die ursprüngliche Idee der Kapsulodese war eine Augmentation des dorsalen Bandapparats, wie etwa in der Operation nach Blatt [12]. Die wahrscheinlich nach wie vor am häufigsten praktizierte Variante der Kapsulodese verwendet einen am Os scaphoideum gestielten Bandstreifen des Lig. intercarpale dorsale [13]. Dieser wird bei der Operation entlang des Faserverlaufs des skapholunären Bandes umgeschlagen und am Os lunatum mittels eines Knochenankers fixiert. Während in den ersten 2 Jahren nach der Operation viele Patienten eine deutliche Verbesserung der Beschwerden erfahren, kommt es jedoch im weiteren Verlauf zu einer zunehmenden Degeneration der Handwurzel sowie einer erneuten Fehlrotation der Knochen. In einer großen Studie konnten wir jedoch zeigen, dass nach 5 Jahren die karpalen Winkel erneut dem präoperativen pathologischen Ausmaß entsprechen [14]. Die Durchführung einer Kapsulodese ist aufgrund der symptomatischen Verbesserung der Patienten eine durchaus mögliche Therapieoption, der Patient muss jedoch über die fortschreitende Degeneration ehrlich aufgeklärt werden.

\section{Tenodesen}

Hier finden i.d.R. Varianten der von Brunelli und Brunelli ursprünglich beschriebenen Tenodese unter Verwendung eines Streifens der Flexor-carpi-radialisSehne Anwendung [15]. Dabei wird ein Bandstreifen von der Palmarseite des Skaphoids durch einen Bohrkanal nach dorsal umgeleitet und hier in Richtung des dorsalen Anteils des skapholunären Bandes am Mondbein fixiert. Moderne Varianten wie etwa die Drei-LigamentTenodese von Garcia-Elias et al. führen hier zusätzlich eine weitere Verstärkung des dorsalen Bandanteils durch [8]. Obwohl zu diesem Operationsverfahren Ergebnisse nur zu einem etwas kürzeren Nachbeobachtungszeitraum vorliegen, unterscheiden sich in einer Studie die Resultate nach 37 Monaten kaum [16]. Vorteil der Methode ist insbesondere eine deutliche Aufrichtung des Skaphoids durch die bessere Kontrolle des distalen Skaphoidpols. Wahrscheinlich kann jedoch auch diese Therapieoption die fortschreitende Degeneration der Handwurzel nicht dauerhaft verhindern. 


\section{Bone-Ligament-Bone-Transplantate}

Ein anderer Therapieansatz ist die Transplantation von 2 Knochenfragmenten, die durch einen straffen Bandanteil zusammengehalten werden [17]. In diesem Fall werden die knöchernen Anteile in das Os scaphoideum sowie Os lunatum eingebracht und fixiert. Selbst bei einer Subselektion von Patienten mit ausschließlich dynamischer Instabilität also einer Patientengruppe mit wahrscheinlich von Haus aus deutlich besserer Prognose - zeigen sich hier im Langzeitverlauf keine befriedigenden Ergebnisse [18]. Vermutlich liegt dies an der fehlenden Kontrolle der Fehlrotation, die durch den relativ schmalen Bandstreifen des Transplantats nicht erzielt werden kann.

\section{Rettungsoperationen}

Die klassischen Rettungsoperationen am Handgelenk sind die Entfernung der proximalen Handwurzelreihe (Proximal Row Carpectomy, PRC) sowie die mediokarpale Teilarthrodese. Aufgrund zunehmender Veränderungen im kapitolunären Gelenkraum ist die Entfernung der proximalen Handwurzelreihe nur in frühen Stadien der Degeneration (SLAC wrist Grad 1 und 2) sinnvoll. Durch beide Operationen können verlässliche und auch langfristig stabile Ergebnisse erzielt werden, die klinisch einer Bandrekonstruktion sehr ähnlich sind [19,20]. Da die mediokarpale Teilarthrodese formell einer Teilversteifung der Handwurzel entspricht, wird das Verfahren von vielen Patienten zunächst zurückhaltend beurteilt, ist aber eine verlässliche $\mathrm{Op}$ tion mit gesichertem Langzeitergebnis.

\section{Zusammenfassung}

Skapholunäre Bandverletzungen sind komplexe Krankheitsbilder, die einer differenzierten Diagnostik und Therapie bedürfen. Ziel der Therapie ist neben einer Verbesserung der Symptome vor allem die Vermeidung von frühzeitig drohenden arthrotischen Veränderungen. Die stadiengerechte Therapie reicht von konservativen Maßnahmen bis zu Versteifungen des Handgelenks und erfordert fundierte Kenntnisse der Biomechanik sowie operative Erfahrung.

\section{Literatur}

${ }^{1}$ Viegas SF, Tencer AF, Cantrell J et al. Load transfer characteristics of the wrist. Part II. Perilunate instability. J Hand Surg Am 1987; 12: 978-985

2 Geissler WB, Freeland AE, Savoie FH et al. Intracarpal soft-tissue lesions associated with an intra-articular fracture of the distal end of the radius. J Bone Joint Surg Am 1996; 78: 357-365

${ }^{3}$ Larsen CF, Mathiesen FK, Lindequist S. Measurements of carpal bone angles on lateral wrist radiographs. J Hand Surg Am 1991; 16: 888-893

${ }^{4}$ Megerle K, Pohlmann S, Kloeters $O$ et al. The significance of conventional radiographic parameters in the diagnosis of scapholunate ligament lesions. Eur Radiol 2011; 21: 176-181

${ }^{5}$ Lawand A, Foulkes GD. The "clenched pencil" view: a modified clenched fist scapholunate stress view. J Hand Surg Am 2003; 28: 414418

${ }^{6}$ Mayer S, Hahn P, Bruckner T et al. Aussagekraft präoperativer MRT-Diagnostik hinsichtlich Läsionen des skapholunären Bandes im klinischen Alltag. Handchir Mikrochir Plast Chir 2013: 45: 26-32

7 O'Meeghan CJ, Stuart W, Mamo $V$ et al. The natural history of an untreated isolated scapholunate interosseus ligament injury. J Hand Surg Br 2003; 28: 307-310

8 Garcia-Elias M, Lluch AL, Stanley JK. Three-ligament tenodesis for the treatment of scapholunate dissociation: indications and surgical technique. J Hand Surg Am 2006; 31: 125134

${ }^{9}$ Cohen MS, Taleisnik J. Direct ligamentous repair of scapholunate dissociation with capsulodesis augmentation. Tech Hand Up Extrem Surg 1998; 2: 18-24

${ }^{10}$ Hom S, Ruby LK. Attempted scapholunate arthrodesis for chronic scapholunate dissociation. J Hand Surg Am 1991; 16: 334-339

${ }^{11}$ Rosenwasser MP, Miyasajsa KC, Strauch RJ. The RASL procedure: reduction and association of the scaphoid and lunate using the
Herbert screw. Tech Hand Up Extrem Surg 1997; 1 : 263-272

12 Blatt G. Capsulodesis in reconstructive hand surgery. Dorsal capsulodesis for the unstable scaphoid and volar capsulodesis following excision of the distal ulna. Hand Clin 1987; 3: 81-102

13 Bickert B, Sauerbier M, Germann G. Scapholunate ligament repair using the Mitek bone anchor. J Hand Surg Br 2000; 25: 188-192

${ }^{14}$ Megerle K, Bertel D, Germann G et al. Longterm results of dorsal intercarpal ligament capsulodesis for the treatment of chronic scapholunate instability. J Bone Joint Surg $\mathrm{Br}$ 2012; 94: 1660-1665

15 Brunelli GA, Brunelli GR. [A new surgical technique for carpal instability with scapho-lunar dislocation. (Eleven cases)]. Ann Chir Main Memb Super 1995; 14: 207-213

${ }^{16}$ Moran SL, Ford KS, Wulf CA et al. Outcomes of dorsal capsulodesis and tenodesis for treatment of scapholunate instability. J Hand Surg Am 2006; 31: 1438-1446

${ }^{17}$ Cuenod P. Osteoligamentoplasty and limited dorsal capsulodesis for chronic scapholunate dissociation. Ann Chir Main Memb Super 1999; 18: 38-53

18 Soong M, Merrell GA, Ortmann FT et al. Longterm results of bone-retinaculum-bone autograft for scapholunate instability. J Hand Surg Am 2013 : 38: 504-508

${ }^{19}$ Saltzman BM, Frank JM, Slikker W et al. Clinical outcomes of proximal row carpectomy versus four-corner arthrodesis for post-traumatic wrist arthropathy: a systematic review. J Hand Surg Eur Vol 2015; 40: 450-457

20 Neubrech F, Mühldorfer-Fodor M, Pillukat T et al. Long-term results after midcarpal arthrodesis. J Wrist Surg 2012; 1: 123-128

\section{Prof. Dr. med. Hans-Günther Machens \\ Chefarzt \\ Priv.-Doz. Dr. med. Kai Megerle \\ Oberarzt}

Klinik und Poliklinik für Plastische

Chirurgie und Handchirurgie

Klinikum rechts der Isar,

Technische Universität München

Ismaninger Straße 22

81675 München

kai.megerle@mri.tum.de 